\title{
Mediación e información
}

Mediation and information

\author{
Oswaldo Francisco de Almeida Júnior \\ Universidade Estadual de Londrina \\ ofaj@ofaj.com.br
}

\section{Resumen}

El trabajo del profesional de la información, en especial el bibliotecario, puede ser definido como la mediación entre la necesidad informacional y informaciones específicas presentes en el universo - casi infinito- informacional. Para que esa mediación se concretice, la acción de ese profesional debe abarcar desde la atención al usuario, el almacenamiento, el tratamiento técnico, hasta la creación de productos documentales, etc. Hace algún tiempo, varios autores defendieron el cambio del paradigma de área, pasando del foco en la colección al foco en la información. Con base en esos presuestos, la investigación "Mediación de la información y múltiples lenguajes" se desarrolla con el siguiente objetivo general: "Analizar la mediación de la información, no sólo en ámbito de su relación más estrecha con la diseminación y el transferencia de la información, sino, también, y principalmente, entendida como base determinante del trabajo bibliotecario y de las implicaciones que acerca de ella refleja sobre el trabajo y conocimiento de los lenguajes presentes en los diversos media informacionales". La investigación tiene por propósito dar las condiciones para que haya una confrontación entre la literatura y la posición de los que realmente actúan en los espacios informacionales. Se defiende aquí la necesidad, o la oportunidad, de pensar el área a partir de la idea de información, pero de manera más rigurosa, norteada hacia la mediación de la información. Sería esta, la mediación, el objeto o, atendiendo las otras maneras de ver la ciencia, el núcleo epistemológico del área. Presenta un concepto provisional de mediación de la información oriundo del proyecto de investigación anteriormente desarrollado por el grupo "Interfaces: información y conocimiento". Como la investigación está en curso, las conclusiones y los resultados todavía no están, obviamente, disponibles.

Palabras clave: Mediación. Información. Lenguajes multimedia. Profesional de la Información. Ciencia de la Información.

\section{Mediación de la información}

La mediación de la información todavía no ocupa el espacio que, nosotros entendemos, se la debe atribuir dentro del quehacer de los profesionales de la información.

\begin{abstract}
The work of the information professional, in special the librarian, can be defined as the mediation between the information need and the specific information which is present in the universe-barely infiniteinformational. For that mediation to become real, the action of that professional should include from user service, storage and technical handling, to the creation of documentary products, etc. For some time, different authors have defended a change of paradigm in the LIS area, moving its focus on the collection to a focus on information. On the basis of this presupposition, the research project "Mediation of information and multiple languages" pretends: "To analyze the mediation of information, not alone in the scope of its narrower relation with the dissemination and transference of information, but, also, and mainly, understood as the determinant backgorund of library work; and analyze the implications of information mediation on the knowledge of the several information media and their processing". The research project aims at establishing the bacground for a confrontation between the literature and the position that actually governs practice in the informational spaces. It is here defended there is a need, or opportunity, to rethink the area from the idea of information, but, in more rigorous way, that is, guided from the concept of information mediation. From this point of view, information mediation would be the object-or, attending to others ways of thinking science, the epistemological nucleus-of area. A provisional concept of mediation of information is presented, resulting from a previous research project developed by the group "Interfaces: information and knowledge". As the research is in course, the conclusions and results are not still available, obviously.
\end{abstract}

Keywords: Mediation. Information. Multimedia languages. Information professional. Information Science.

El discurso de estos profesionales señala al carácter esencial de las actividades relativas a la mediación de la información. Sin embargo, la práctica desnuda la forma verdadera como la entienden, o sea, una acción que se puede ejer- 
cer por cualquier profesional, independientemente de su formación.

Un gran problema que estorba la oferta eficaz de los servicios por las bibliotecas es el hecho de que el Servicio de Referencia e Información no es considerado como espacio privilegiado de las actividades de los bibliotecarios. En él, así entiende el sentido común, pueden actuar todos los tipos de profesionales, incluyendo los no preparados o no formalmente aptos para ejercer actividades ligadas a la mediación, difusión, transferencia, propagación de la información.

El discurso que clava la biblioteca como espacio transformador o como polo desencadenador de transformaciones y cambios desentona, así, totalmente de la practica diaria de las bibliotecas y del quehacer bibliotecario.

Bibliotecas públicas, escolares, universitarias y también las bibliotecas de las empresas utilizan casi siempre a empleados no cualificados para atender a sus públicos. Muchos aprendices, alumnos de cursos de graduación de Biblioteconomía ejercen esta función. En las bibliotecas de las escuelas, como es de amplio conocimiento una vez que las discusiones sobre el tema fueron y siguen siendo parte de muchos eventos del área-, a los profesores reajustados por diversas razones, se les obliga a dejar el aula, y optan por desarrollar sus actividades en ellas, satisfaciendo un papel para el cual no están, en la mayoría de las veces, preparados para ejercer. Cabe recordar que ésta no es una mera posición corporativista, sino que se apoya en la idea o presupuesto de que existe la necesidad de una formación ajustada para el ejercicio de las actividades vinculadas a la biblioteca escolar. Lo que invariablemente sucede es que los profesores, siguiendo el sentido común implantan, con excepciones, por supuesto, un modelo de biblioteca con el cual habían tenido contacto, es decir, una biblioteca que se preocupa con la ampliación del acervo cuanto más grande el número de libros, mejor es la biblioteca-, que prepara técnicamente los materiales sin una noción clara del porqué lo están haciendo, y que ofrece como servicios los préstamos, la consulta o una u otra acción de lectura. Aunque tienen conocimientos de políticas pedagógicas que sostienen sus actividades en el aula, estos profesores tienen dificultades para transportar tales políticas para el hacer de la biblioteca escolar.

En el caso de la biblioteca pública la situación es incluso peor. A pesar de estar dirigidas y supervisadas por profesionales capacitados, en este caso, los bibliotecarios, los ayudantes que trabajan en el Servicio de Referencia desconocen, en la mayoría de las veces, las actividades desarrolladas en algunos sectores de la biblioteca. Así actúan con base, solamente, en un quehacer aislado, en acciones desarticuladas, basándose en un entrenamiento rápido, que privilegia una acción mecánica y rutinaria. Los bibliotecarios están en número pequeño, escaso para hacer frente a la demanda de información. La situación se mantiene toda vez que la gran mayoría de los usuarios de ese tipo de biblioteca está formada por estudiantes que buscan ese espacio para realizar investigaciones solicitadas por los profesores de los cursos básicos, medios e, incluso, universitarios.

Muchos son los autores que afirman que los estudiantes son la mayoría, tal vez alcanzando más del $80 \%$ de los usuarios de la biblioteca pública.

En base a textos e investigaciones de la década de 1990, incluyendo los realizados por el autor, Oswaldo Francisco de Almeida Júnior (1997, p.28), lidiando con porcentaje, defiende que "El porcentaje exacto de la participación de los estudiantes en el total de usuarios de las bibliotecas públicas, difícilmente se podría obtener, aunque es posible [...] establecerlo entre 80 el y 90\%".

Las bibliotecas de las universidades buscan actuar de manera distinta, poniendo profesionales bibliotecarios para los servicios de atendimiento al público. Incluso así, se obliga a contar con el trabajo de ayudantes. Cabe a aquellos profesionales, a los bibliotecarios, la tarea de la administración, no específicamente del sistema de información presente en aquel espacio, sino del día a día de la biblioteca, de los aspectos burocráticos. Por carencia absoluta de tiempo de los bibliotecarios, a los ayudantes se les encarga la mayoría de las actividades relacionadas al atendimiento de los usuarios. En el Servicio de Referencia e Información, por ejemplo, el número de bibliotecarios puestos allí es escaso para proveer las demandas que proceden de las necesidades diarias de los alumnos. Vale recordar que estas necesidades están constituidas, en gran parte, por la búsqueda de libros indicados como básicos por los profesores.

En el caso de las bibliotecas especializadas, incluso, considerando que los trabajos desarrollados por ellas están volcados básicamente hacia la mediación de la información, hay una cuota del atendimiento que es efectuado por ayudantes, incluidos entre éstos, los aprendices.

Una división por tipo de biblioteca, usada tradicionalmente por el área y aquí utilizada, no se ajusta, siendo hoy cuestionada en demasía. Sin embargo, nos valemos de ella para demostrar 
solamente la indiferencia o el pequeño interés del área en relación a los trabajos dirigidos hacia el público.

Defendemos que hay una distinción básica entre las actividades vinculadas al atendimiento de los usuarios entre los cuatro tipos de biblioteca. En el caso de los tres primeros tipos, o sea, bibliotecas escolares, públicas y universitarias, estas actividades se dirigen hacia la indicación e intermediación de las fuentes que potencialmente pueden atender a una determinada necesidad. A su vez, las actividades de las bibliotecas empresariales (o especializadas, centros de información, y otras denominaciones generalmente usadas mencionándose e identificando, total o parcialmente, aquellas bibliotecas) están focalizadas en la entrega de informaciones que atiendan y satisfagan las necesidades de sus usuarios. Además, otro diferencial de importancia extrema es el hecho de que los usuarios, en el caso de estas últimas bibliotecas, son un número pequeño y la mayoría conocidos. La rotación y la inclusión de nuevos usuarios no son normalmente cuantitativamente significativas, al contrario de lo que ocurre en las bibliotecas escolares, públicas y universitarias.

\subsection{Mediación de la información como objeto del área}

Sin embargo, defendemos una tesis relacionada con un concepto más amplio de la mediación. Ella no estaría restringida solamente a las actividades relacionadas directamente al público, sino con todas las acciones del profesional bibliotecario, en todo el quehacer de ese profesional. La mediación estaría presente, de manera no explícita en la selección y elección de los materiales que formarán parte del acervo de la biblioteca, en todo el trabajo de procesamiento técnico, en las actividades de desarrollo de colecciones y, también en el servicio de referencia e información. Presente en todas esas acciones, la mediación sería parte del propio objeto del área de la información. Específicamente, en lo referente al área de la Ciencia de la Información, su objeto volvería a ser más la mediación que la información. Muchas son las áreas que tienen a la Información como su objeto de estudio, de análisis, de preocupación. Lo que distingue el área de la Ciencia de la Información de las otras áreas que se ocupan, también de la información, sería el hecho de que el objeto de la Ciencia de la Información no es la información en si misma, sino la mediación de ella.

Con base en ese presupuesto, realizamos una investigación llamada "La mediación de la información: orientadora del quehacer biblioteca- rio", iniciada en el 2002 y finalizada en febrero del 2005. En ella, buscamos conocer como los profesionales del área entienden el concepto de mediación de la información. Se hizo claro en las manifestaciones de la mayoría de los profesionales, que a la mediación se la entiende de manera localizada, restringida a los espacios donde hay un acercamiento directo entre los usuarios y la biblioteca. El espacio donde se da la mediación fue apuntado, invariablemente, como el de servicio de referencia e información, consolidando el sentido común en el tema.

La investigación que, sin embargo, desarrollamos, se propone profundizar los aspectos teóricos relativos al tema de la mediación, siendo extendidos los estudios ya iniciados, en especial los concernientes a los conceptos de mediación presentes en las obras de Vygosthy, Martin Barbero, Gilberto Velho y Marx. Además de ellos, también se propone ampliar el análisis de los autores que se ocupan de la mediación de la lectura, incluyendo las discusiones sobre las diferencias de esta con la animación de lectura, término utilizado para las actividades desarrolladas en bibliotecas públicas.

Entre los resultados obtenidos con referencia a la mediación de la información, se resaltan las ideas de "Ponte" y de "Elo", muchas veces manifestadas por los profesionales auscultados. En las explicaciones de los conceptos presentados por ellos, queda obvia la relación de los términos con una pasividad, con una pretensa neutralidad del profesional de la información en el momento en el cual ejerce actividades vinculadas al atendimiento de los usuarios.

\subsection{Neutralidad y parcialidad}

Esa idea, sin embargo esperada, se presentó de modo muy acentuado en las respuestas y en el discurso del profesional, involucrando ideas que nos llevan a concepciones enraizadas en el área, vinculas a conceptos de neutralidad e imparcialidad.

La parcialidad no es exclusiva del Servicio de Referencia e Información, por el contrario, se la puede detecta en todos los trabajos desarrollados en la biblioteca. Eso ocurre porque la biblioteca lidia con la información, que depende del usuario para concretizarse, y porque actúa con personas que, evidentemente, se distinguen una de las otras, con intereses, propósitos, posturas, ideas, concepciones individuales. La mediación busca ajustar, de la mejor manera, la necesidad del usuario con las informaciones que irán a satisfacer tales necesidades. Si los intereses y necesidades son individuales, las informaciones se deben identificar con ellos, destacándose de 
un universo infinito para atender a la solicitud de un usuario específico. El propio hecho de que el acceso al universo informacional sea irrealizable, de que los mecanismos de búsqueda sean limitados y de que abarquen una cuota del todo informacional, ya confirma la idea de parcialidad de la mediación. Además, la información depende del mercado editorial; debe someterse a censuras que vienen de intereses de grandes industrias, de concepciones de los mecenas de investigaciones científicas, de los mantenedores de instituciones y órganos de investigaciones, de los dueños de empresas que actúan con la información, de los grupos de poder presentes en el área académica, etc. Incluso con la facilidad de vehicular ideas vía red electrónica de datos, la información buscada es aquella disponible en sitios legitimados por la tradición de las instituciones o personas que las mantienen. Así, la construcción de un acervo o la búsqueda de informaciones es determinada por una parcialidad existente incluso antes de la acción de la biblioteca.

Del mismo modo, la neutralidad no puede estar presente en las acciones desarrolladas por las bibliotecas, en especial en las bibliotecas públicas. Aunque se intente alcanzar esa neutralidad, la idea de mediación ya presupone la imposibilidad de lograr ese objetivo. Para que la biblioteca pudiera ser neutra, tendría que ofrecer al usuario todas las informaciones vinculadas a la cuestión que desencadena el proceso de referencia. Estamos lejos de concretizar la idea de la biblioteca universal perseguida por los hombres desde la antigüedad. Si un espacio, incluso virtual, pudiera tornar realidad ese sueño anhelado por el hombre, aún así no se obtendría la neutralidad en los trabajos desarrollados por la biblioteca, pues otros factores contribuyen a su inexistencia, como la dificultad en exteriorizarse la cuestión verdadera, los problemas para la interacción entre bibliotecario y usuario, los procedimientos arraigados, no cuestionados y tenidos como correctos en el quehacer bibliotecario, etc.

\subsection{Concepto de mediación de la información}

Basada en estas ideas y otras, la búsqueda anterior determinó un concepto de mediación de la información, aun en carácter embrionario, aunque permita su empleo y uso, que busca disociar esa nueva visión de la mediación —no restricta al Servicio de Referencia e Información y si configurando en el objeto del área- de aquella presente en el sentido común de los profesionales del área. Tal concepto sería expresado así:
"Mediación" de la Información es toda la acción de interferencia —realizada por el profesional de la información-, directa o indirecta; conciente o inconsciente; individual o colectiva; que propicia la apropiación de la información que atienda, plena o parcialmente, una necesidad informacional.

\subsection{Interferencia}

Un punto importante que debe de ser destacado en ese concepto es la idea de interferencia. Como resultado de los datos levantados, del contacto y análisis de la literatura y de las discusiones y debates realizados en el grupo de investigación, una certidumbre quedó evidenciada: la de que las acciones del profesional de la información no son neutras, no son imparciales y resultan siempre en una interferencia.

La interferencia se contrapone a la idea de aislamiento, pasividad. El espacio informacional se constituye al mismo tiempo en objeto y sujeto de la historia, del destino de la sociedad. Es objeto, pues recibe influencias y es sujeto en todos los momentos en que influencia en lo que interfiere. La unidad informacional, no es un espacio aislado, ajeno y exento de interferencias. Todas las informaciones sociales, de una o otra forma, influyen y exigen posturas y cambios tanto del espacio informacional, como de los que en ellos actúan y de los servicios implantados y ofrecidos. Sin embargo, parece no ser esa, exactamente, la forma como los espacios informacionales entienden su relación con la sociedad y con las transformaciones de esta.

El artículo de Depallens (1987) se presenta como de suma importancia para la discusión de esa relación, ya que, abordando las bibliotecas de Nicaragua, constata que, a diferencia de otras instituciones, las bibliotecas publicas, como ejemplo de espacio informacional, no sufrieron ninguna alteración tras la Revolución Sandinista. Los mismos trabajos, las mismas actitudes y posturas, la misma acción, en fin, los mismos objetivos antes y después de la Revolución que derrumbó a Somoza y que alteró por completo la vida del país. Tal constatación puede ser transportada al Brasil, aunque no haya ocurrido una revolución (en verdad, el golpe de 1964 fue una contra-revolución) pero, el periodo anterior a 1964, durante la dictadura militar, tras la "apertura política", y, más recientemente, la "transición democrática", durante todos esos períodos de la vida nacional, durante esos momentos conflictivos, antagónicos y de situaciones socio-políticas y económicas diferentes, la biblioteca pública en el Brasil, a ejemplo de la de Nicaragua, tuvo poca o casi ninguna alteración. Los mismos trabajos, las mismas posturas, 
la misma actuación. Lo mismo ocurrió y ocurre en la mayoría de los países del mundo.

Retomando el concepto de mediación de la información, es menester que se reafirme la importancia de la interferencia en el quehacer del profesional de la información. Incluso buscando la imparcialidad $-\mathrm{y}$ sabiendo que ella nunca será alcanzada- el profesional debe preocuparse con la relación muy cercana entre interferencia y manipulación.

Esta, sí debe ser totalmente abolida de las acciones del profesional, sin embargo en el trabajo cotidiano, aunque sea inconscientemente, todo profesional esta sujeto a enfrentarse a ella en su quehacer. La línea que separa la interferencia de la manipulación es extremadamente tenue. A pesar de eso, el profesional de la información debe buscar el difícil equilibrio sobre esa línea.

La interferencia en el quehacer del profesional de la información niega la postura enfáticamente defendida, de que ese profesional es pasivo, superveniente, destituido de una aptitud proactiva, sin iniciativa, que sólo contribuye, auxilia y apoya.

El profesional de la información, así, pasa a ser entendido en otra esfera, en otro estrato profesional, la de aquellos que hacen historia, son sujetos en la sociedad y participan efectivamente en la construcción del destino de la humanidad. Incluso más que eso: es con la conciencia de que interfiere, de que se realiza como profesional en la relación con el usuario, que la democracia de la información podrá concretizarse, porque la democracia presupone peleas y embates entre ideas, concepciones, valores, etc., diferentes; peleas y embates de formas y maneras distintas de explicar el mundo; peleas y embates por el poder; porque la democracia es el espacio en que esas peleas son trabadas. Del mismo modo, lidiando con la información, los espacios informacionales permiten y propician esas peleas y embates, transformándose en los ámbitos aptos para la realización, efectivación y concretización de la democracia, de la inclusión informacional y social.

\subsection{Servicios}

Lo que distingue los espacios informacionales, en la medida en que aceptamos la idea de que la mediación determina todo el quehacer del profesional de la información, es el servicio ofrecido, los servicios proporcionados a los usuarios y que tiene como objetivo permitir la apropiación de la información o de informaciones que satisfacen una determinada necesidad.
La mayoría de las bibliotecas públicas, escolares y hasta las universitarias, ofrecen como servicio el uso domiciliar de materiales y su uso dentro del espacio asignado dentro de la propia biblioteca. Esos son los dos grandes y únicos servicios ofrecidos. Pocas son las bibliotecas que ofrecen algo diferente de esos dos servicios. Esos servicios lidian, casi invariablemente, con el soporte libro.

A mediados de la década de 1990, mucho se discutió sobre el cambio de paradigma de la Biblioteconomía. Las discusiones eran válidas no sólo para la Biblioteconomía, sino para toda el área de la Ciencia de la Información. Se defendió que el área vivía la alteración de un paradigma del acervo hacia un paradigma de la información. El paradigma del acervo centraba toda la acción de los profesionales que actuaban en las bibliotecas en los soportes informacionales, en especial en el libro. A pesar de la creencia en el cambio de paradigmas, todo lleva a creer que esa transformación no se concretizó o fue realizada parcialmente y en pequeños nichos del área.

El paradigma del acervo se opone a las actividades ligadas a la información, pues la perocupación, en ese caso, es permitir y posibilitar el acceso físico a los soportes informacionales. En esa perspectiva, el profesional poco se preocupa con el contenido de los materiales, llevándolo a una postura tecnicista, en que la técnica tiene su razón en ella misma. Muchas de las críticas dirigidas al profesional de la información, en especial al bibliotecario, deben ser acreditadas a la idea de neutralidad y al apego al paradigma del texto escrito, de la palabra impresa en la diseminación de la información.

Bajo el paradigma de la información, todos los soportes son objeto de interés y preocupación del quehacer y de las acciones de los profesionales de la información.

Acompañando al multimedia, cuatro son los segmentos que determinarían los tipos de soportes de la información: el texto escrito, la imagen fija, la imagen en movimiento y el sonido. Todos ellos pasan a ser parte de los intereses de todos aquellos que lidian con la información, en especial los que poseen como núcleo básico de actuación, la mediación de la información.

Aún en el ámbito virtual, los servicios ofrecidos en los espacios informativos miran los soportes y no la información. En las bibliotecas públicas, por ejemplo, hay hoy una preocupación en permitir el acceso de todos a la Internet. Disponer la Internet para uso de los que frecuentan la biblioteca es normalmente entendida - por las corrientes que creen que es la biblioteca el es- 
pacio privilegiado de democratización de la información- como la mejor y más importante actividad que posibilita la relación de la población con la información. La idea de que la Internet democratiza la información puede y debe ser entendida como volcada a la defensa de un tipo de pensamiento que no corresponde al de la diseminación. Al contrario, propalar que la Internet democratiza la información significa decir que la diseminación puede ocurrir dentro o a partir de un vehículo, y no de una acción. O mejor, que la acción presente en el trabajo ligado a la Internet es exacta y únicamente el ofrecimiento de la máquina, del ordenador y de las herramientas que posibilitan la entrada y el uso de la Internet (Almeida Júnior, 1998).

Muchos propagan la idea de que en la Internet están todas las informaciones producidas en el mundo. Es obvio que tal afirmación no pasa de ser una gran utopía. Representa, a lo mucho, el deseo de quién la disemina. La información, a ejemplo de lo que discutimos en relación a las acciones del profesional de la información, no es neutra, imparcial, apolítica; no está aislada, volando sola sobre los soportes que la buscan aprisionar. La información sólo se concretiza en la interacción con aquel o aquellos que la necesitan e, incluso así, relacionándose con el motivo que determinó su búsqueda. Se equivoca quién piensa que la información es neutra o imparcial, principalmente porque quedan olvidadas las implicaciones políticas, sociales, económicas, culturales etc., existentes tras cada información. Pensando en el cotidiano de las unidades de información, la idea de que la lnternet acumula todas las informaciones producidas en el mundo lleva, invariablemente, al uso exclusivo de esa red como instrumento y herramienta de recuperación de información. ¿Por qué buscar una información en otro lugar si, con absoluta seguridad, está presente en la Internet? Quien pierde una vez más es el usuario.

Esa idea parece llevar a la Internet a ser una candidata que se junta a los dos grandes servicios ofrecidos por las bibliotecas (préstamos domiciliares y consultas locales), pues, como ellos, será elegida por constituirse en un espacio de almacenaje de informaciones y que cuenta con productos y mecanismos de recuperación. No hay necesidad, para que de ella se haga uso, de una acción diseminadora, de una mediación. El quehacer bibliotecario, en esos servicios, se hace de forma disimulada, escondido tras la neutralidad e imparcialidad de las técnicas.

El mismo pensamiento, la misma suposición ocurre en relación a los soportes textuales, a la palabra impresa. Se cree que es ella la única capaz de transmitir, de diseminar informaciones y conocimiento. A pesar de que vivimos en una sociedad letrada en que la información y el conocimiento son transmitidos prioritariamente por el texto escrito, hay otras formas por las cuales las personas pueden apropiarse de ellos. Entre esas formas, además de los varios tipos de soportes diferenciados del texto escrito, hay también la forma oral que, en un país con un alto índice de analfabetos y analfabetos funcionales, debe ser considerada como una de las maneras de ser mediada la información, bajo pena de excluir del acceso a la información a un inmenso contingente de brasileños.

Pocos son los espacios informativos que se preocupan y utilizan otros soportes distintos del libro, del periódico o que contengan la palabra escrita. Eso ocurre, en buena medida, porque varios de esos soportes necesitan de accesorios para que de ellos se haga uso. Además de eso, sin embargo, el desconocimiento, la "no alfabetización" en los lenguajes de esos medios representa y explica mejor cómo son entendidos en los espacios informativos.

Conocer los lenguajes de los múltiples medios que sostienen informaciones potenciales y de ellas hacer uso como recursos y fuentes en la mediación de la información es uno de los principales desafíos del área de la información en la contemporaneidad.

\section{Nuevo objeto}

Hay hoy en día, en el área de Biblioteconomía y de la Ciencia de la Información, una preocupación con la búsqueda de caminos que indiquen el norte, de reflexiones que apunten rumbos futuros para el área.

Esa, evidentemente, no es una necesidad exclusiva de la Biblioteconomía y de segmentos afines -como la Archivología y la Museología, ambas entendidas como subáreas de la Ciencia de la Información-, pero se hace presente dentro de un contexto de crisis de las profesiones y de definiciones de límites, de fronteras entre los varios campos del trabajo y del conocimiento humano.

Tales discusiones y reflexiones apuntan para una reordenación, reorientación, redefinición de los intereses, de los espacios de actuación, de los límites restrictivos de cada campo de trabajo y del conocimiento humano.

Las herramientas automatizadas, las nuevas tecnologías de la información, las facilidades por parte de determinados segmentos de la sociedad de acceso a la información, las rápidas y variadas transformaciones, no sólo en el ámbito 
de las ciencias, sino también en las áreas sociales, políticas, culturales, etc., determinan y exigen nuevas posturas, nuevos contornos de actuación, interés, preocupación, interacción, relación, de todos los campos del conocimiento humano. La Biblioteconomía y la Ciencia de la Información, inmersas en esa coyuntura que tiene a la transformación y al cambio como diferenciadores en relación a situaciones anteriores, también se ven impelidos, de forma compulsiva, a repensarse, proponiendo nuevas formas de articularse con la sociedad y con las otras áreas. El carácter interdisciplinario de ellas, presupone una constante e interminable discusión interna sobre su objeto de estudio y su ámbito de actuación. Hay momentos, como el actual, en que esa discusión se hace más apremiante y exige respuestas, y que éstas sean coherentes con las perspectivas futuras.

Sin embargo, parece existir una contradicción entre la necesidad de estar en constante cambio, acompañando las transformaciones sociales, y lo que efectivamente se concretiza en el área de la Biblioteconomía.

Las herramientas y los instrumentos empleados por el área, las acciones desarrolladas en los espacios en que ella se hace concreta, se realiza, indican y apuntan a una propuesta de mantenimiento, de solidificación de una realidad, de una situación; casi siempre son adversas y repudian cambios y transformaciones.

Ese panorama contraría los discursos presentes y diseminados por los teóricos y por los profesionales del área, y se opone a la exigencia actual de una posición efectiva y clara a favor de los cambios, bajo pena de, si no se procede así, tornarse inútil como área que responde a los a anhelos y necesidades de la sociedad.

La investigación desarrollada por nosotros, tiene como interés prioritario la propuesta de análisis de una vertiente mediadora como base del entendimiento de las concepciones que fundamentan específicamente el área de la biblioteconomía y explican el quehacer y las acciones de los profesionales que en ella actúan. La principal hipótesis con la cual trabajamos -y que entendemos deba transformase en tesis al final de la investigación - se orienta a -aún hoy y a pesar de un discurso que apunta para otra idea- la idea de que la Biblioteconomía, hoy, está sostenida por conceptos, instrumentos y herramientas que defienden, no claramente, formas dirigidas a la preservación. La diseminación de la información - poco como servicios ofrecidos a los usuarios y menos aún como base teórica-, incluso en un momento en que la literatura afirma ser la información, en contraposición al acervo, el paradigma de la Biblioteconomía y de la propia Ciencia de la Información, es asumida por los profesionales sólo en el ámbito del discurso. La práctica de la información, el quehacer del profesional de la información, dentro del que nos posibilita la observación, todavía son orientados y diseccionados para el texto impreso, la palabra escrita, dejando de lado soportes que sostienen otros medios. Esa situación se debe a la dificultad de obtener los equipos imprescindibles y necesarios para hacer uso de esas medios, pero, principalmente, por el desconocimiento y la "no alfabetización", tanto del profesional como del usuario, en el lenguaje de esos medios.

\section{Objetivos}

El objetivo general del proyecto está así enunciado: Analizar la mediación de la información, no solo en el ámbito de su relación mas estrecha con la diseminación y la transferencia de la información, sino, también, y principalmente, entendida como base determinante del quehacer bibliotecario y las implicaciones que sobre ella inciden el trabajo y el conocimiento de los lenguajes presentes en los varios medios informativos.

En relación a los objetivos específicos, éstos son: cuestionar y discutir los motivos por los cuales las acciones volcadas al trabajo de transferencia y de diseminación de la información son considerados, en el espacio de las unidades de información, como de importancia secundaria; detectar en la literatura del área, como son recuperadas, analizadas, entendidas, asimiladas y practicadas las acciones volcadas para el uso de medios diferenciados del texto impreso y de la palabra escrita; levantar y analizar cuáles son los profesionales que actúan en el área y que registran de alguna manera sus acciones, los principales servicios ofrecidos por las unidades de información empleando medios diferenciados y cómo se da la relación de ellas con el público atendido; identificar dentro de la actuación del profesional de la información, cuáles son los momentos y en qué medida la mediación de la información de medios diferenciados se hace presente; cuestionar a los profesionales de la información sobre su conocimiento de los lenguajes de los medios presentes en los multimedia (texto escrito, imagen fija, imagen en movimiento y sonido); cuestionar a los profesionales de la información sobre su conocimiento del lenguaje virtual y cómo se emplea y qué peso se le da en los espacios en que actúa; $y$, finalmente, identificar, a partir de las respuestas de los profesionales y de la literatura, el conocimiento por parte del usuario de los lenguajes 
de los diferentes medios y cómo es su relación con esos lenguajes.

\section{Procedimientos metodológicos}

La investigación surgió de un amplio análisis de la literatura, identificando materiales que pudiesen contribuir con el tema enfocado. Preferentemente, los textos deberían estar concentrados o con interés en la actuación de los espacios informativos, con énfasis en las bibliotecas y en los bibliotecarios brasileños. Evidentemente, el trabajo de lectura y la búsqueda de nuevos textos irá a repercutir en toda la investigación.

El próximo paso será el contacto con los profesionales que actúan en las unidades de información brasileñas, utilizando desde el correo electrónico hasta contactos personales, sea directamente en los espacios donde actúan o en eventos específicos del área. A esos profesionales, cuya muestra ya está siendo definida, les será aplicado un cuestionario que, todavía sin un análisis muy riguroso, deberá permitir, mediante cuestiones abiertas, que se conozca interpretaciones, entendimientos, visiones, etc., de los asuntos básicos y de interés de la investigación.

La idea principal es posibilitar a los profesionales de la información, un espacio para que puedan presentar, vehicular, exteriorizar sus conceptos, sus entendimientos de forma que sus opiniones sean oídas, sean tomadas en cuenta y tengan ellos una real participación en los destinos, rumbos y caminos del área de la información.

El cuestionario será estructurado teniendo como base la búsqueda por conocer las opiniones y conceptos de los profesionales sobre lo que está basada teóricamente su acción. También buscará conocer cómo el profesional ve su actuación y cómo ocurren - si ocurren- trabajos, actividades y servicios con medios diferenciados del texto escrito. Se buscará conocer como se da la relación de los usuarios con los diferentes medios y su grado de conocimiento, empatía e intimidad con los lenguajes de esos medios. Se buscará instigar una reflexión de los profesionales sobre su acción, principalmente en el ámbito de la mediación de la información, sobre el empleo de los varios lenguajes multimedia.

La investigación tiene por propósito dar condiciones para que haya un confronto entre la literatura y la posición de los que efectivamente actúan en los espacios informativos. Los estudios teóricos se distancian, buena parte de las veces, de las situaciones concretas en las cuales los profesionales del área están envueltos, y tal distanciamiento colma por tornar los resulta- dos de esos estudios en poco aplicables o perdidos y olvidados en documentos e informes. La implicación y la participación de los profesionales de la información en esta investigación -es la pretensión del grupo de investigación- será mucho más amplia que meramente objetos de análisis.

Las respuestas obtenidas a partir de los cuestionarios serán tabuladas y analizadas; y la síntesis servirá como base para las discusiones que ocurrirán dentro de un grupo de investigación "Interfaces: información y conocimiento", grupo que ya existe desde 1998 y que actualmente se dedica a investigaciones dirigidas a la mediación de la información.

Los varios lenguajes serán subdivididos y quedarán bajo la responsabilidad de los profesores que componen el grupo de pesquisa. Alumnos de iniciación científica, becados o no, y colaboradores tendrán, dentro de las necesidades de la investigación, la responsabilidad por subtemas, supervisados y orientados por los profesores.

Finalmente, con los datos colectados, resultantes de la aplicación de los cuestionarios para la muestra de los profesionales que actúan en las unidades de información, se hará un confronto con las discusiones y reflexiones oriundas de las ideas rescatadas de la literatura y de la propuesta de análisis del área a partir de las concepciones de mediación, reconociendo la interrelación entre los tres puntos. La investigación se completa con la presentación de los resultados de ese confronto.

\section{Referencias}

Almeida Júnior, Oswaldo Francisco de (1998). Ágora informacional. // Ciencias de la Información. 29:4 (Out./Dez. 1998).

Almeida Júnior, Oswaldo Francisco de (1995). Biblioteca pública: ambigüidade, conformismo e ação guerrilheira do bibliotecário. São Paulo: APB, 1995. 10p. (Ensaios APB, 15).

Almeida Júnior, Oswaldo Francisco de (2003). Biblioteca pública: avaliação de serviço. Londrina: EDUEL, 2003.

Almeida Júnior, Oswaldo Francisco de (1997). Bibliotecas públicas e bibliotecas alternativas. Londrina: EDUEL, 1997.

Almeida Júnior, Oswaldo Francisco de (2004a). Informação pública: conceitos e espaços. // Valentim, Marta (Org). Atuação profissional na área de informação. São Paulo: Polis, 2004. 71-81.

Almeida Júnior, Oswaldo Francisco de (2004b). Implicações entre formação e objeto da área de informação. // Encuentro de Directores, 8. y Encuentro de Docentes de Escuelas de Bibliotecología y Ciencias de la Información del Mercosur, 6. 30 de agosto a 1 de setiembre de 2004, Mar del Plata, Argentina. Anais... Mar del Plata, 2004. (CD-ROM)

Almeida Júnior, Oswaldo Francisco de (2004c). Mediação da informação. // Fadel, Bárbara (Org.). A informação nas organizações sociais: desafios em face de multipli- 
cidade de enfoques. Marília: FUNDEPE, 2004c. (CDROM)

Almeida Júnior, Oswaldo Francisco de (2004d). Profissional bibliotecário: um pacto com o excludente. // Baptista, Sofia Galvão, Müeller, Suzana Pinheiro Machado (Orgs.). Profissional da informação: o espaço de trabalho. Brasília: Thesaurus, 2004d. 70-86.

Almeida Júnior, Oswaldo Francisco de (1997). Sociedade e Biblioteconomia. São Paulo: Polis/APB, 1997.

Barreto, Aldo de Albuquerque (1994). A questão da informação. São Paulo em Perspectiva. 8:4 (Out./Dez. 1994) 3-8.

Braga, Maria de Fátima Almeida (2002). Meios e modos de apropriação da informação e do conhecimento. // Castro, César Augusto (Org.) (2002). Ciência da Informação e Biblioteconomia: múltiplos discursos. São Luis: EDUFMA, 2002. 109-119.

Breton, Philippe (1999). A manipulação da palavra. São Paulo: Loyola, 1999.

Briggs, Asa; Burke, Peter (2004). Uma história social da mídia. Rio de Janeiro: Jorge Zahar, 2004.

Burke, Peter (2003). Uma história social do conhecimento. Rio de Janeiro: Jorge Zahar, 2003.

Canclini, Néstor Garcia (1995). Consumidores e cidadãos: conflitos multiculturais da globalização. Rio de Janeiro: Editora UFRJ, 1995.

Celadec (1984). Definição e conteúdo da documentação popular. Recife: SEDIPO, 1984. (Mimeografado).

Chartier, Roger (2002), A mediação editorial. Chartier, Roger (2002). Os desafios da escrita. São Paulo: Editora UNESP, 2002. p.61-76.

Chaui, Marilena (2006). Cultura e democracia: o discurso competente e outras falas. 11.ed. São Paulo: Cortez, 2006.

Chaui, Marilena (2006). Simulacro e poder: uma análise da mídia. São Paulo: Editora Fundação Perseu Abramo, 2006.

Coelho Neto, José Teixeira (1996). Do paradigma do acervo ao paradigma da informação. // Anais do I Simpósio Brasil Sul de Informação, Londrina, 1996. Londrina: UEL, 1996. 15-30.

Depallens, Jacques (1987). La bibliotecologia necesita de una revolución cultural. // Revista Interamericana de Bibliotecologia. 10:1 (Ene/Jun. 1987) 7-14.
Dizard Júnior, Wilson (1998). A nova mídia: a comunicação de massa na era da informação. Rio de Janeiro: Jorge Zahar Editor, 1998.

Duarte, Newton (2003). Sociedade do conhecimento ou sociedade das ilusões? Campinas: Autores Associados, 2003.

Dupas, Gilberto (2000). Ética e poder na sociedade da informação. São Paulo: Editora da UNESP, 2000.

Freire, Paulo (2005). Pedagogia do oprimido. 43.ed. Rio de Janeiro: Paz e Terra, 2005.

Martin-Barbero, Jesús (1997). Dos meios às mediações: comunicação, cultura e hegemonia. Rio de Janeiro: Editora UFRJ, 1997.

Martin-Barbero, Jesús (2003). Globalização comunicacional e transformação cultural. Moraes, Denis de (Org.) (2003). Por uma outra comunicação: mídia, mundialização cultural e poder. Rio de Janeiro: Record, 2003. 57-86.

Mattelart, Armand (2001) História da sociedade da informação. São Paulo: Loyola, 2001.

Meszáros, Istvan (2005). A educação para além do capital. São Paulo: Boitempo, 2005.

Moraes, Denis de (Org.) (2003). Por uma outra comunicação: mídia, mundialização cultural e poder. Rio de Janeiro: Record, 2003.

Morin, Edgar (2003). Uma mundialização plural. // Moraes, Denis de (Org.) (2003). Por uma outra comunicação: mídia, mundialização cultural e poder. Rio de Janeiro: Record, 2003. 349-366.

Oliveira, Marlene; Araújo, Eliany Alvarenga de (2002). Os paradigmas da biblioteconomia e da ciência da informação e os novos contextos de informação. // Castro, César Augusto (Org.) (2002). Ciência da Informação e Biblioteconomia: múltiplos discursos. São Luis: EDUFMA, 2002. 35-49.

Ramonet, Ignácio (2003). O poder midiático. // Moraes, Denis de (Org.) (2003). Por uma outra comunicação: mídia, mundialização cultural e poder. Rio de Janeiro: Record, 2003. 243-252.

Velho, Gilberto; Kuschnir, Karina (Org.) (2001). Mediação, cultura e política. Rio de Janeiro: Aeroplano, 2001. 